\title{
Dark matter induced Brownian motion
}

\author{
Ting Cheng ${ }^{1,3, a}{ }_{\mathbb{C}}$, Reinard Primulando ${ }^{2, \mathrm{~b}}$, Martin Spinrath $^{1, \mathrm{c}} \mathbb{C}_{\mathbb{C}}$ \\ ${ }^{1}$ Department of Physics, National Tsing Hua University, Hsinchu 30013, Taiwan \\ 2 Department of Physics, Center for Theoretical Physics, Parahyangan Catholic University, Jl. Ciumbuleuit 94, Bandung 40141, Indonesia \\ ${ }^{3}$ Present Address: Max-Planck-Institut für Kernphysik, Heidelberg, Germany
}

Received: 25 February 2020 / Accepted: 22 May 2020 / Published online: 10 June 2020

(C) The Author(s) 2020

\begin{abstract}
We discuss a novel approach for directional, light dark matter searches inspired by the high precision position measurements achieved in gravitational wave detectors. If dark matter interacts with ordinary matter, movable masses are subject to an effect similar to Brownian motion induced by the scattering with dark matter particles which exhibits certain characteristics and could be observed. We provide estimates for the sensitivity of a hypothetical experiment looking for that motion. Interestingly, if successful, our approach would allow to constrain the local distribution of dark matter momentum.
\end{abstract}

\section{Introduction}

Cosmological and astrophysical data provides overwhelming evidence for dark matter (DM). Unfortunately, this data does not tell us anything definite about the nature of DM itself.

To solve this riddle, there has been tremendous experimental effort in the last decades focussing, in particular, on theoretically well-motivated weakly interacting $\mathrm{TeV}$ scale $\mathrm{DM}$, so called Weakly Interacting Massive Particles (WIMPs). These efforts remain until today without any conclusive evidence for a discovery. For that reason, recently other potential mass regions for DM are more seriously considered and new ideas are developed to test them experimentally, see, e.g. [1]. In this paper we follow this line and discuss a novel experimental approach for light DM. As we will see our method would work, in principle, to very small masses. However, under more realistic assumptions, its sensitivity lies in the range above about $10 \mathrm{MeV} / \mathrm{c}^{2}$.

Our proposal is motivated by the great achievements in laser interferometry for gravitational wave detectors, but as

\footnotetext{
a e-mail: ting.cheng@mpi-hd.mpg.de

be-mail: rprimulando@unpar.ac.id

c e-mail: spinrath@phys.nthu.edu.tw (corresponding author)
}

we will see later LIGO and other current earth-bound gravitational wave detectors are not well suited for our method.

We are not the first to propose to use gravitational wave detectors or interferometers in general as DM detectors, see for instance [2-11]. Nevertheless, our approach is very different from theirs. They usually focus on very light DM with masses well below $1 \mathrm{keV} / \mathrm{c}^{2}$, where DM behaves more like a classical field with a very long wave-length. In our method the particle nature of DM is essential. In fact, it is loosely inspired by the work of one of the authors [12]. Interestingly, results of the KWISP detector were presented which is looking for dark energy particles with an opto-mechanical setup [13] somewhat similar to our proposal. There has also been a search for very heavy DM using displacement sensors [14].

The idea of this paper is based on the picture that any movable target with mass $M_{T}$, is actually in a bath of DM. If DM has some interaction with ordinary matter, that will induce some random motion of the target, which is conceptually similar to Brownian motion. We call this here Dark Brownian Motion (DBM) which can in principle be observed. It is experimentally easier to study the DBM if the target is constrained to a certain volume, e.g., if it is a pendulum. The concrete system is not relevant here, but we assume that the motion of the target in absence of any external forces can be understood well. In the following section, we will discuss the details of our method.

\section{The detection method}

Since the earth has a relative velocity to the DM bath we expect that the DBM has a preferred direction. This directional dependence is well-known for directional dark matter detection with more conventional detectors [15]. In particular, we adopt here the formalism for the DM recoil momentum spectrum as it was discussed, for instance, in [16]. To 
keep the discussion simple, we will assume that the DM interaction with ordinary matter is fully elastic. The total event rate, $R$, is given by

$$
\begin{aligned}
R= & (Z+N)^{2} \sigma_{\mathrm{DM}-N} \frac{M_{T}}{M_{\mathrm{mol}}} \frac{\rho_{\mathrm{DM}}}{M_{\mathrm{DM}}} \bar{v}_{\mathrm{DM}} \\
= & 0.37\left(\frac{Z+N}{12} \frac{\sigma_{\mathrm{DM}-N}}{10^{-31} \mathrm{~cm}^{2}} \frac{M_{T}}{10^{-3} \mathrm{~g}}\right. \\
& \left.\times \frac{\rho_{\mathrm{DM}}}{0.3 \mathrm{GeV} / \mathrm{cm}^{3}} \frac{20 \mathrm{MeV}}{M_{\mathrm{DM}}} \frac{\bar{v}_{\mathrm{DM}}}{341 \mathrm{~km} / \mathrm{s}}\right) \frac{1}{\mathrm{~s}},
\end{aligned}
$$

where $Z$ and $N$ are the atomic number and the number of neutrons respectively, $\sigma_{\mathrm{DM}-N}$ is the average cross section of DM with a target nucleon, $M_{T}$ the target mass, $M_{\text {mol }}$ is the molar mass of the target, $\rho_{\mathrm{DM}}=0.3 \mathrm{GeV} / \mathrm{cm}^{3}$ [17] is our assumed local DM energy density, $\bar{v}_{\mathrm{DM}}=\int \mathrm{d}^{3} v_{\mathrm{DM}} v_{\mathrm{DM}} f\left(\vec{v}_{\mathrm{DM}}\right)$ is the average local DM velocity. We would like to point out here already, that although the interaction rate has units of inverse time it does not correspond to a well-defined frequency since the hits occur at random intervals with random recoil momenta. In fact, due to the lack of periodicity the Fourier transformation of the DM hit signal usually has no dominant peak around the average hit rate, but rather many random small peaks scattered over all frequencies. Therefore, we perform our analysis in the time-domain and not in the frequency-domain.

We assume that DM couples dominantly to nucleons in an isospin conserving manner, and throughout the paper we assume ${ }^{12} \mathrm{C}$ as target material motivated by the use of graphene as reflective material [18] and tunable oscillators [19] which might be able to give us a light, tunable mirror in a laboratory size interferometer. Actually, the physics in this letter is not related to (laser) interferometers. We only require a precise measurement of the position and/or velocity of a macroscopic object. To our knowledge laser interferometers are the best technology for that purpose and therefore we will consider them as an example. Other potential technologies could be, for instance, magnetic resonance force microscopy, see [20] and references therein or optically levitated microspheres, cf. [14]. Therefore, we encourage the reader to be open-minded and consider our proposal independently from that.

For the DM velocity distribution we assume the Standard Halo Model (SHM)

$f_{\mathrm{gal}}(\mathbf{v})= \begin{cases}\frac{N}{\left(2 \pi \sigma_{v}\right)^{3 / 2}} \exp \left(-\frac{|\mathbf{v}|^{2}}{2 \sigma_{v}^{2}}\right) & \text { if }|\mathbf{v}|<v_{\mathrm{esc}}, \\ 0 & \text { if }|\mathbf{v}|>v_{\mathrm{esc}},\end{cases}$

where $v_{\mathrm{esc}}=550 \mathrm{~km} / \mathrm{s}, \sigma_{v}=220 \mathrm{~km} / \mathrm{s}$ [17] and for the translation between the galactic rest frame and the local laboratory frame we used the formulas in Appendix A of [21].

It is not clear that the SHM is a good description of the DM velocity distribution. In a recent work [22], for instance, it was argued that some fraction of the DM could receive a significant shift towards larger velocities opening up direct detection constraints to a lower mass region. Similarly, the DM velocity profile does not need to be isotropic and there could be streams of DM passing through the solar system, see, for instance, [23]. A higher DM velocity and/or anisotropy could make our setup more sensitive. Nevertheless, a detailed study of such possibilities goes beyond the scope of the current work and to make our results more easily comparable to other studies, we will follow the conventional SHM assumption.

Under these assumptions and the formalism of [16] we can simulate the distribution of recoil momenta. For the incoming momenta we picked random values following the SHM distribution. The recoil momenta are then fully fixed by choosing random values for the scattering angles, $\theta$ and $\phi$, which we assumed to have a flat distribution in $\cos \theta$ and $\phi$. This allows us to evaluate the asymmetry parameter

$A=\frac{N_{+}-N_{-}}{N_{+}+N_{-}}=p_{+}-p_{-}$,

where $N_{ \pm}$is the number of events of the DM hitting the detector with positive/negative target recoil momentum $q_{R}$ with $\left|q_{R}\right|>q_{\min }$ along the axis we are probing. The parameter $q_{\min }$ is a cutoff parameter which we will discuss later in more detail. We have also introduced here the likelihoods $p_{ \pm}=N_{ \pm} /\left(N_{+}+N_{-}\right)$.

To determine $A$ we simulated for each parameter point one million scattering events to get the distribution of the recoil momenta, from which we can derive $p_{ \pm}$easily. We estimate the statistical uncertainty for $A$ using ordinary error propagation with the $1 \sigma$ uncertainty of the number of events given by $\sigma_{ \pm}=\sqrt{N_{ \pm}}$, and find

$\sigma_{A}^{2}=\frac{4}{R \Delta t} p_{+} p_{-}$

with $\Delta t$ as the length of data taking, which we have fixed for the rest of the paper to be $10 \mathrm{~min}$.

At this point we want to sketch how the counting of events could be performed. We mentioned already that we assume to have a mathematical model for the experiment which predicts the position and velocity of the target mass in the absence of forces. Additionally, we also assume that the time resolution of the detector can resolve the DM hits. Suppose now the data is taken in time bins $t_{1}, t_{2}, \ldots$ and the target is hit by DM particle at some time between $t_{h}$ and $t_{h+1}$. The target position and velocity at $t_{h+1}$ will then differ from its predicted values given by the values measured at the previous time bins, and from the differences we can reconstruct the recoil momentum of the DM hit. For a harmonic oscillator, this can be done straight-forwardly using a Runge-Kutta algorithm as we convinced ourselves. In fact, for the asymmetry we only 


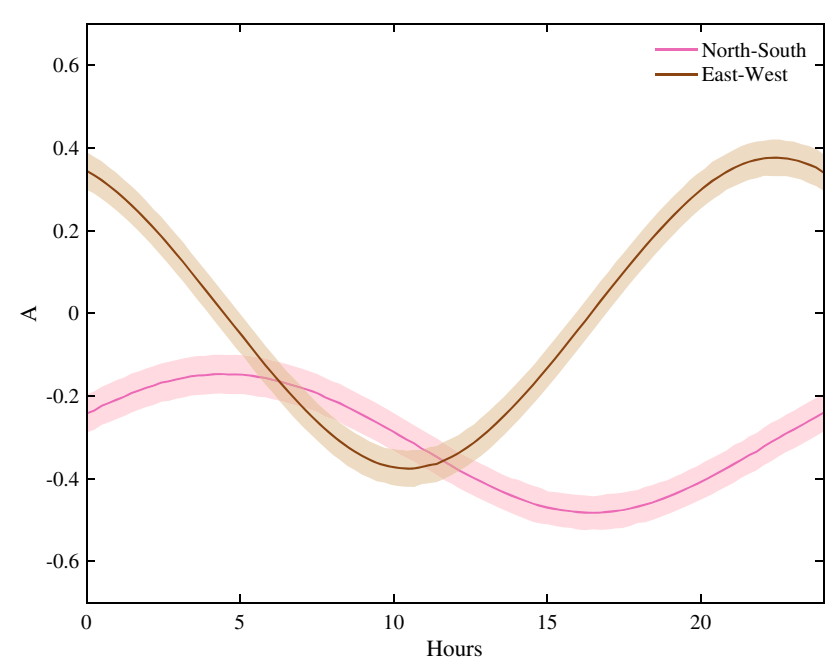

Fig. 1 The expected time dependence of the asymmetry $A$ in northsouth (pink) and east-west direction (brown) in the earth frame within $24 \mathrm{~h}$ in Hsinchu on June 1st. The colored bands represent the estimated $1 \sigma$ uncertainty. For more details see main text

really need to reconstruct the sign of the recoil momenta, which is comparatively easy.

While in this paper we will use the value of $A$ from 10 minutes of hypothetical data taking, it is interesting to see how the value of $A$ changes over time due to the change of the relative orientation of the detector. In Fig. 1 we plot the time dependence of the asymmetry for an imaginary experiment located in Hsinchu within 24 h on June 1st, 2020. For the error bars we have assumed that we take data for ten minutes with a target mass of $10^{-3} \mathrm{~g}$ and $\sigma_{\mathrm{DM}-N}=10^{-31} \mathrm{~cm}^{2}$.

Figure 1 also contains some information about the directional dependence of the asymmetry parameter. The northsouth direction has the largest absolute value of $A$, and the east-west direction has a larger value of daily modulation of $A$. There is some room for optimization here by rotating the experiment, in terms of the absolute value or the daily modulation of the asymmetry. However for simplicity, in the rest of the paper, we choose the east-west alignment of our hypothetical experiment. Moreover we simulate the data at $10 \mathrm{pm}$ local Hsinchu time. Then $A \approx 0.37, \bar{v}_{\mathrm{DM}} \approx 341 \mathrm{~km} / \mathrm{s}$ and the relative velocity of the lab with respect to the DM halo along this direction is $v_{\text {lab,EW }} \approx-183 \mathrm{~km} / \mathrm{s}$. A positive $A$ here means that there are more hits expected with a recoil momentum in the eastern direction.

We would like to clearly emphasize at this point, that we propose here to count hits which then enter in the determination of $A$. The hit rates in a certain direction should be modulated with a frequency of roughly $1 /$ day and 1 /year translating into a modulation of $A$, but we do not propose to measure these frequencies directly. One of the major reasons to consider, nevertheless, $A$ as the relevant observable, and not just the total number of events or the measured momentum distri-

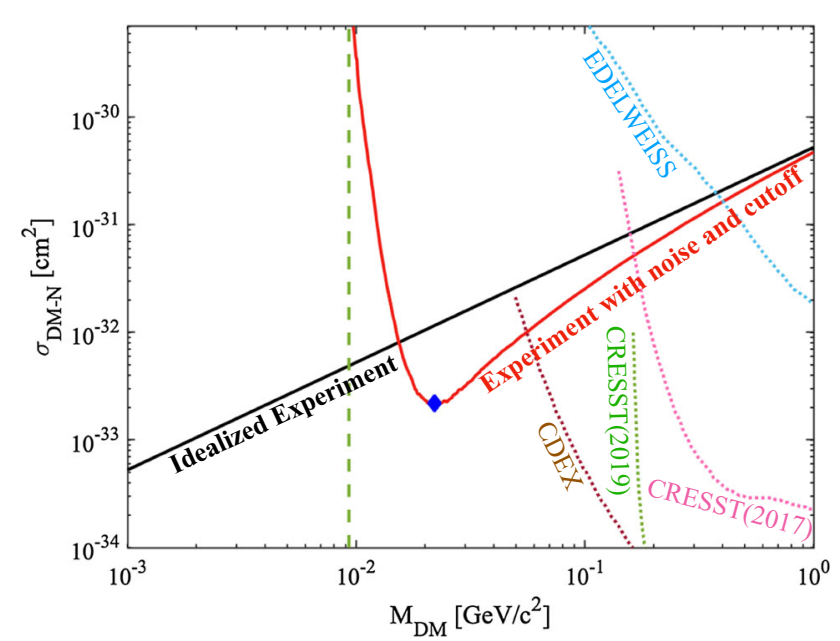

Fig. 2 The estimated $2 \sigma$ sensitivity of our experimental setup for a total target mass of $10^{-3} \mathrm{~g}$. The black line is for an idealized experiment and the red line includes our noise estimates a minimal momentum cutoff, $q_{\text {min }}=2 \times 10^{-23} \mathrm{~kg} \mathrm{~m} / \mathrm{s}$. We also show the exclusion bounds from CRESST [24,25], EDELWEISS (Migdal) [26] and CDEX (Migdal) [27] as pink, green, light blue and dark red dotted lines, respectively. The blue diamond corresponds to the blue diamond in Fig. 3. For more details, see main text

bution itself, is its characteristic of a more pronounced time dependence, which can be a powerful discrimination from backgrounds. Moreover, it is more robust against uncertainties of the recoil momentum measurement after applying a momentum cutoff as will be introduced later.

\section{Estimated sensitivity}

In this section we will provide some simple sensitivity estimates to illustrate the physics behind it and what kind of precision and background reduction would be needed to observe DBM and, in particular, use it as a competitive constraint compared to more conventional DM searches.

In Fig. 2 we show our estimate for the sensitivity. We begin our discussion with an idealized experiment with infinitely precise momentum measurement and no background events. For a total target mass of $M_{T}=10^{-3} \mathrm{~g}$ we find the black line as $2 \sigma$ exclusion level, i.e. $\langle A\rangle / \sigma_{A}=2$. The dependence of this line can be easily understood. For smaller masses, the DM flux, and hence the event rate, increases while the shape of the recoil momentum distribution remains the same, i.e.

$\frac{\langle A\rangle^{2}}{\sigma_{A}^{2}}=R \Delta t \frac{\left(p_{+}-p_{-}\right)^{2}}{4 p_{+} p_{-}}$.

In this formula the only explicit dependence on the DM mass and the DM-nucleon cross section is in $R$, cf. Eq. (2.1). 
In reality though there will be experimental uncertainties which alter the sensitivity. To illustrate their potential impact we introduce two sources of uncertainty.

The first is a generic background giving a recoil momentum of the test masses. That could be seismic noise, nearby traffic, radioactivity, etc. Many of them can be reduced by choosing the location and materials of the experiment, e.g. putting it underground, choosing low radioactivity materials, and using a suspension system to reduce seismic noise. Furthermore, as already mentioned many of these backgrounds could be easily discriminated from a potential DM signal due to the different characteristic time-dependencies of the asymmetries which we do not explicitly consider in this section though. Nevertheless, we want to discuss some estimates for two particle backgrounds which are definitely present as examples. Those are neutrinos and hits from residual air molecules. The largest neutrino flux on earth is from the solar $p p$ neutrinos with $\Phi_{v} \approx 6 \times 10^{10} \mathrm{~cm}^{-2} \mathrm{~s}^{-1}$ at an energy of about $0.4 \mathrm{MeV}$ corresponding to a nucleon cross section to carbon of about $10^{-44} \mathrm{~cm}^{2}$. For our assumed target mass of $10^{-3} \mathrm{~g}$ this corresponds to a neutrino event rate of $\mathcal{O}\left(10^{-14}\right) \mathrm{s}^{-1}$. Obviously, even including all other neutrino sources this is negligibly small compared to the DM event rate, we are considering here, cf. Eq. (2.1).

The differential event rate of air molecules hitting the target is

$\frac{\mathrm{d} N}{\mathrm{~d} t \mathrm{~d} v}=n A|v| f(v)$

where $A$ is the surface of the target, $v$ the velocity of the air molecules in the direction probed by the experiment, $f(v)$ its standard Maxwellian distribution and $n=P /\left(k_{B} T\right)$ is the number density of the molecules with $P$ the pressure, $k_{B}$ the Boltzmann constant and $T$ the temperature. Note that the target is assumed to be symmetric and can be hit from both sides. The hit rate after integrating over all velocities is hence,

$$
\begin{aligned}
\frac{\mathrm{d} N}{\mathrm{~d} t} & =\frac{P}{k_{B} T} A \sqrt{\frac{2 k_{B} T}{\pi m}}=P A \sqrt{\frac{2}{\pi m k_{B} T}} \\
& \approx 8.3 \times 10^{9}\left(\frac{P}{10^{-10} \mathrm{mbar}} \sqrt{\left.\frac{20 \mathrm{~K}}{T} \frac{A}{\mathrm{~mm}^{2}}\right) \frac{1}{\mathrm{~s}}} .\right.
\end{aligned}
$$

Here we have assumed that the residual gas consists of molecular hydrogen which is usually dominant in ultrahigh vacuum, cf. e.g. [28], since vacuum pumps can remove heavier molecules more efficiently. For the pressure, we assume a vacuum system like in the LHC [29] or advanced LIGO [28] and for the temperature a cryonic system as in KAGRA [30]. The target surface is assumed to be $1 \mathrm{~mm}^{2}$. We want to remind the reader, that lower pressures and temperatures have been achieved experimentally and there can be room for improvement, but as a conservative estimate we use numbers from current similar experiments if possible.

Now that poses a problem at first, since this rate is much larger than a realistic DM hit rate. Let us assume in the following that the data bins are $\delta t=0.1 \mathrm{~ns}$ long, which is common in optical experiments. Then we expect about one hit from residual molecules in one data bin.

Our approach is still feasible, in principle, since the distribution of recoil momenta from DM and the atmosphere are different. For this let us first calculate the recoil momentum distribution per data bin. The differential recoil momentum is

$\frac{\mathrm{d} q_{\mathrm{atm}}}{\mathrm{d} t}=q \frac{\mathrm{d} N}{\mathrm{~d} t}=2 m n A v|v| f(v) \mathrm{d} v$

where we assume that each molecule just bounces off the target, $q=2 m v$. The expectation value is obviously zero (we neglect any potential airflow in the chamber). The variance of the Gaussian distribution is then

$$
\begin{aligned}
\sigma_{q_{\mathrm{atm}}}^{2} & =\left\langle q_{\mathrm{atm}}^{2}\right\rangle=4 m^{2} n A \delta t \int v^{2}|v| f(v) \mathrm{d} v \\
& =4 m^{2} n A \delta t\left[\sqrt{\left.\frac{8}{\pi}\left(\frac{k_{B} T}{m}\right)^{3 / 2}\right]}\right. \\
& =8 \sqrt{\frac{2}{\pi}} P A \delta t \sqrt{m k_{B} T} \\
& \approx 6.1 \times 10^{-48}\left(\frac{P}{10^{-10} \mathrm{mbar}} \frac{A}{\mathrm{~mm}^{2}} \frac{\delta t}{0.1 \mathrm{~ns}} \sqrt{\frac{T}{20 \mathrm{~K}}}\right) \mathrm{kg}^{2} \mathrm{~m}^{2} / \mathrm{s}^{2}
\end{aligned}
$$

From data bin to data bin we hence expect a momentum uncertainty of order of $\sigma_{q_{\mathrm{atm}}} \approx 2.5 \times 10^{-24} \mathrm{~kg} \mathrm{~m} / \mathrm{s}$. A cut on the recoil momentum can therefore suppress the fluctuations induced by the residual gas hits significantly and we can still hope to see a DM signal as long as the DM recoil momentum spectrum allows for larger recoil momenta. In every realistic experiment, we would expect anyway such minimal resolution of the recoil momentum and that brings us to the second of the experimental uncertainties.

The second major deviation from an ideal experiment is the uncertainty of the position and/or the recoil momentum measurement. Considering a harmonic oscillator with an eigenfrequency $\omega_{0}$ and a position resolution $d_{\min }$ as a classical toy model, this implies a minimal momentum resolution of the order of $q_{\min }=M_{T} \omega_{0} d_{\min }$. To improve the sensitivity one could imagine to split the detector mass into smaller detector cells, which would lower $q_{\text {min }}$ linearly with the number of cells. As sensitivity goal we use a rather naive interpretation of the LIGO resolution $d_{\min }=10^{-19} \mathrm{~m}$ at a frequency of $\omega_{0}=100 \mathrm{~Hz}$ [31] which leads to a minimal momentum 
resolution of the order of $\mathcal{O}\left(10^{-23}\right) \mathrm{kg} \mathrm{m} / \mathrm{s}$. For definiteness we will use in the following $q_{\min }=2 \times 10^{-23} \mathrm{~kg} \mathrm{~m} / \mathrm{s}$ unless specified otherwise. This number corresponds to the maximum recoil momentum for DM with a mass of a few $\mathrm{MeV}$ with an order one uncertainty from the velocity distribution so that we can expect to potentially see a signal for DM masses above that. Furthermore, with this choice the background from the residual air molecules is very efficiently suppressed and we end up with an effective rate

$R_{\mathrm{atm}}^{\mathrm{cut}} \approx 5 \times 10^{-6} \mathrm{~Hz}$

Applying this cut makes the experiment almost free from that noise.

LIGO with its $40 \mathrm{~kg}$ mirrors has a much larger momentum cutoff and cannot resolve the momentum recoil of an individual light DM hit or the many hits of air molecules. One might also wonder, if a similar resolution could be achieved anytime soon in an experiment with much lighter mirrors as we require. For instance, the so-called standard quantum limit in gravitational wave detectors [32] is proportional to $1 /\left(M_{T} \omega^{2} L^{2}\right)$, where $L$ is the arm length. Lighter target masses could hence be a problem. But looking at membranes with eigenfrequencies in the $\mathrm{kHz}$ - or even $\mathrm{MHz}$-range excited by DM hits instead, cf. [33] can compensate for that lighter masses. A more thorough study of such matters will be presented elsewhere [34]. Nevertheless, the effect of DBM is independent of laser interferometers. And as we had stated at the beginning of this section already the numbers here should be seen as a target sensitivity for which our suggested method could become competitive to conventional direct DM searches, cf. Fig. 2 and even reach lighter DM masses.

From that point of view our suggestion should be much more sensitive to heavier DM since the expected recoil momentum would be larger. Nevertheless, current constraints on the DM cross section to ordinary matter and the local DM number density suggest that our approach is not competitive to conventional experiments in that mass range.

The uncertainty in the momentum measurement affects recoil momenta larger than $q_{\text {min }}$ as well, but in the asymmetry $A$ only the sign of the momentum enters which we know well, after we discarded all events with recoil momenta smaller than $q_{\text {min }}$.

A momentum cutoff $q_{\min }>0$ on one hand reduces the number of events in the signal region and thus potentially lowers the significance. On the other hand, it can enhance $A$ and the two effects are competing with each other.

In Fig. 2 we show the final estimated sensitivity curve including the noise from residual air molecules and the momentum resolution cutoff as the red line given by the condition $\frac{\langle A\rangle^{2}}{\sigma_{A}^{2}+\sigma_{\mathrm{bkg}}^{2}}=4$

where $\langle A\rangle$ and $\sigma_{A}$ include both of the DM signal and the background while $\sigma_{\text {bkg }}$ only includes the hits from the residual gas.

First of all, we can see in Fig. 2 that the momentum cutoff makes it impossible for our hypothetical experiment to discover DM masses less than $\mathcal{O}(10) \mathrm{MeV} / \mathrm{c}^{2}$ (the vertical dashed line is at $9.3 \mathrm{MeV} / \mathrm{c}^{2}$ ). This will be clear from the kinematical arguments which we discuss later. But we can also see that for the mass range above $15 \mathrm{MeV} / \mathrm{c}^{2}$ the momentum cutoff increases the theoretical sensitivity compared to the idealized experiment which is due to the increase in the asymmetry and the very efficient background rejection.

From Fig. 2 it is also clear, that the $q_{\text {min }}$ plays the equivalent role of the energy threshold in conventional DM detectors. In our case though, this depends only indirectly on the chosen target material, instead it depends dominantly on the resolution of the interferometer and the target mass neglecting potential excitation modes of the target material.

To give an impression on how our sensitivity compares to experimental results, we also show the exclusion lines from CRESST [24,25], EDELWEISS (Migdal) [26] and CDEX (Migdal) [27] which are relevant for DM masses above $50 \mathrm{MeV} / \mathrm{c}^{2}$. Cosmology also provides bounds, but they are rather weak. Refs. [35,36], for instance, find that the DMnucleon cross section over the whole displayed mass region should be less than $\mathcal{O}\left(10^{-28}\right) \mathrm{cm}^{2}$.

It is instructive to have a closer look at the momentum cutoff $q_{\text {min }}$. We can easily understand from the most extremal case that the DM asymmetry $A$ can increase for an increasing cutoff. Let $v_{\text {lab }}$ be the lab velocity with respect to the galactic halo in the direction we are probing. For $\left|v_{\text {esc }}-v_{\text {lab }}\right|<$ $q_{\min } /\left(2 M_{\mathrm{DM}}\right)<\left|v_{\text {esc }}+v_{\text {lab }}\right|$ the asymmetry is extremal, $|A|=1$, due to simple kinematics and ignoring any potential backgrounds. This leads to another characteristic feature of a DM signal. Increasing $q_{\mathrm{min}}$ the modulus of the DM induced asymmetry continuously increases until no events are left for an idealized experiment. This is the dashed green line in Fig. 3, where we have set $\sigma_{\mathrm{DM}-N}=2.2 \times 10^{-33} \mathrm{~cm}^{2}$, $\mathrm{M}_{\mathrm{DM}}=22 \mathrm{MeV} / \mathrm{c}^{2}$ which ends at $q_{\min }=6.1 \times 10^{-23} \mathrm{~kg} \mathrm{~m} / \mathrm{s}$. There is of course a trade-off. A larger $q_{\text {min }}$ leads to a larger DM asymmetry, but it will also lead to a decreased DM event rate.

This will additionally be affected by the background from residual gas. We make here the same assumptions as we have used in Fig. 2 and for the cross section and the dark matter mass we use the same numbers as for the idealized experiment here. From the solid orange line in Fig. 3 we see that a very small $q_{\text {min }}$ the observed asymmetry would drop to zero since the number of background events is many orders of magnitude larger than the DM events pushing the asymmetry 


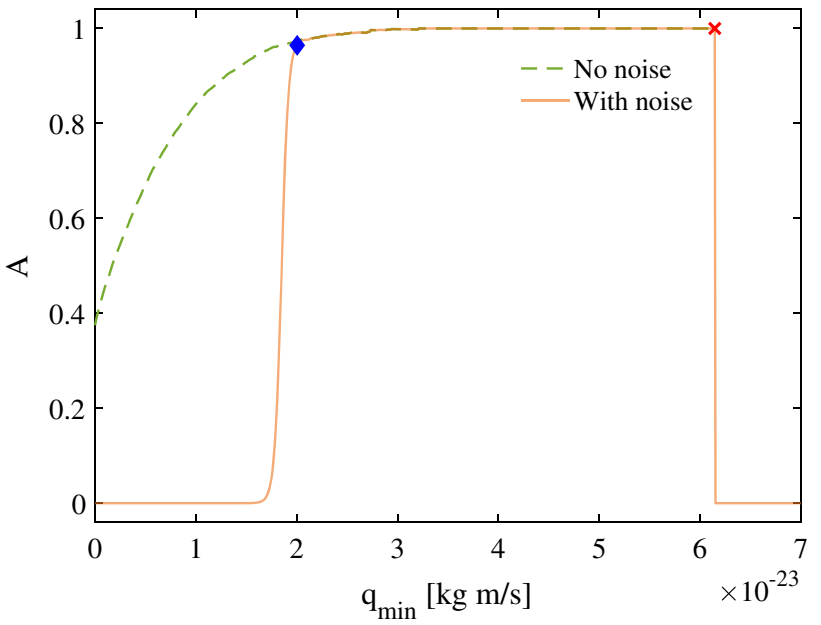

Fig. 3 The dependence of the asymmetry $A$ on a lower bound for the recoil momentum $q_{\min }$ for a benchmark point with (solid line) and without noise from residual gas (dashed line). As soon as $q_{\mathrm{min}}$ is larger than the maximal DM recoil momentum there are no events left and the lines end at the red cross. The blue diamond corresponds to the blue diamond in Fig. 2. For more explanations, see main text

to zero. We also see that the threshold for which this background becomes small is very sharp since the background distribution is comparatively narrow compared to the DM recoil momentum distribution. Note that in theory we still expect an extremely tiny number of background events from the very tail of the distribution such that $A$ drops again to zero above the maximum DM recoil momentum. Varying $q_{\min }$ in the data analysis hence might reveal a previously unidentified background or signal, if it exhibits such features.

Although in our simplified sketch setup we did not consider that possibility explicitly there might be backgrounds which have a broader recoil momentum distribution than the potential DM signal leading to another source of events above the DM cutoff. That could be cosmic rays or radioactive decays in the chamber, which are difficult to model realistically without specifying the location, materials, shielding, etc. which goes beyond the scope of this paper. Generally speaking this would reduce the sensitivity, shifting the sensitivity curve upwards since the DM asymmetry would be washed out. To reduce such backgrounds, one can introduce an upper bound on the recoil momentum $q_{\max }$ in the data analysis.

A careful study of the dependence of the measured asymmetry $A$ on both, $q_{\min }$ and $q_{\max }$, hence might help to understand the background much better in the absence of a signal. Should a potential signal be identified the variation of both would provide valuable information on the recoil momentum spectrum of background and signal. In combination with the information on the time dependence that would be very exciting and would allow to constrain the DM mass scale and halo model.

\section{Summary and conclusions}

In this paper we have discussed the effect of Dark Matter induced Brownian motion which could be used as a novel way to search for light DM. This motion has a characteristic, time- and direction-dependent asymmetry of the recoil momenta due to the relative motion of the earth to the DM halo. This asymmetry and, in particular, its daily modulation could in principle be observed in an experiment, which measures the position/velocity of a target object to very high precision. Currently, interferometers seem to be most promising for that purpose, but not LIGO and other earth-bound telescopes since their mirror masses imply a momentum cutoff well above our expectations for light DM. In the future though, gravitational wave detectors might be able to detect vibrational modes of thin mirrors excited by DM scatterings [33].

Here we have assumed a hypothetical, different kind of experiment (which does not even have to be based on laser interferometers) with lighter target masses. If this experiment can resolve recoil momenta as small as discussed in our paper one could test DM masses down to the $\mathcal{O}(10) \mathrm{MeV} / \mathrm{c}^{2}$ region. For our example, we find that for a DM mass of $22 \mathrm{MeV} / \mathrm{c}^{2}$ one could test a DM-nucleon cross section down to $2.2 \times 10^{-33} \mathrm{~cm}^{2}$ with only 10 minutes of data taking. Further research regarding the feasibility of this numbers considering different experimental approaches is needed in the future.

Our results also depend on other uncertainties like the DM velocity distribution and modifications of that by cosmic ray scatterings or local streams might enhance or weaken considerably the sensitivity of such an experiment.

Acknowledgements We would like to thank Yue-Lin Sming Tsai and Ulises Saldaña-Salazar for useful comments on the manuscript. We also thank the EPJ $\mathrm{C}$ referee for their valuable suggestions and comments. TC and MS are supported by the Ministry of Science and Technology (MOST) of Taiwan under Grant Number MOST 107-2112-M-007-031MY3.

Data Availability Statement This manuscript has no associated data or the data will not be deposited. [Authors' comment: This is a theoretical study and there is no experimental data.]

Open Access This article is licensed under a Creative Commons Attribution 4.0 International License, which permits use, sharing, adaptation, distribution and reproduction in any medium or format, as long as you give appropriate credit to the original author(s) and the source, provide a link to the Creative Commons licence, and indicate if changes were made. The images or other third party material in this article are included in the article's Creative Commons licence, unless indicated otherwise in a credit line to the material. If material is not included in the article's Creative Commons licence and your intended use is not permitted by statutory regulation or exceeds the permitted use, you will need to obtain permission directly from the copyright holder. To view a copy of this licence, visit http://creativecomm ons.org/licenses/by/4.0/.

Funded by $\mathrm{SCOAP}^{3}$. 


\section{References}

1. J. Alexander et al.,. arXiv:1608.08632 [hep-ph]

2. C.J. Riedel, Phys. Rev. D 88(11), 116005 (2013). arXiv:1212.3061 [quant-ph]

3. Y.V. Stadnik, V.V. Flambaum, Phys. Rev. Lett. 114, 161301 (2015). arXiv:1412.7801 [hep-ph]

4. A. Arvanitaki, S. Dimopoulos, K. Van Tilburg, Phys. Rev. Lett. 116(3), 031102 (2016). arXiv:1508.01798 [hep-ph]

5. Y.V. Stadnik, V.V. Flambaum, Phys. Rev. A 93(6), 063630 (2016). arXiv:1511.00447 [physics.atom-ph]

6. A. Branca et al., Phys. Rev. Lett. 118(2), 021302 (2017). arXiv:1607.07327 [hep-ex]

7. C.J. Riedel, I. Yavin, Phys. Rev. D 96(2), 023007 (2017). arXiv:1609.04145 [quant-ph]

8. S. Jung, C.S. Shin, Phys. Rev. Lett. 122(4), 041103 (2019). arXiv:1712.01396 [astro-ph.CO]

9. A. Pierce, K. Riles, Y. Zhao, Phys. Rev. Lett. 121(6), 061102 (2018). arXiv:1801.10161 [hep-ph]

10. S. Morisaki, T. Suyama, Phys. Rev. D 100(12), 123512 (2019). arXiv:1811.05003 [hep-ph]

11. H. Grote, Y.V. Stadnik, Phys. Rev. Res. 1, 033187 (2019). arXiv:1906.06193 [astro-ph.IM]

12. V. Domcke, M. Spinrath, JCAP 1706, 055 (2017). arXiv:1703.08629 [astro-ph.CO]

13. S. Arguedas Cuendis et al., Phys. Dark Univ. 26, 100367 (2019). arXiv:1906.01084 [hep-ex]

14. A. Kawasaki, Phys. Rev. D 99(2), 023005 (2019). arXiv:1809.00968 [physics.ins-det]

15. D.N. Spergel, Phys. Rev. D 37, 1353 (1988)

16. P. Gondolo, Phys. Rev. D 66, 103513 (2002). arXiv:hep-ph/0209110

17. M.C. Smith et al., Mon. Not. R. Astron. Soc. 379, 755 (2007). arXiv:astro-ph/0611671

18. E. Carrasco, M. Tamagnone, J. Perruisseau-Carrier, Appl. Phys. Lett. 102, 104103 (2013)

19. C. Chen, S. Lee, V.V. Deshpande, G.-H. Lee, M. Lekas, K. Shepard, J. Hone, Nat. Nanotechnol. 8, 923 EP (2013)
20. A.L. Barabanov, O.A. Titov, Phys. Rev. C 99(4), 045502 (2019). arXiv:1810.09896 [nucl-th]

21. F. Mayet et al., Phys. Rept. 627, 1 (2016). arXiv:1602.03781 [astroph.CO]

22. T. Bringmann, M. Pospelov, Phys. Rev. Lett. 122(17), 171801 (2019). arXiv: 1810.10543 [hep-ph]

23. C.A.J. O'Hare, C. McCabe, N.W. Evans, G. Myeong, V. Belokurov, Phys. Rev. D 98(10), 103006 (2018). arXiv:1807.09004 [astroph.CO]

24. G. Angloher et al., CRESST collaboration. Eur. Phys. J. C 77(9), 637 (2017). arXiv:1707.06749 [astro-ph.CO]

25. A.H. Abdelhameed et al., CRESST collaboration. Phys. Rev. D 100(10), 102002 (2019). arXiv: 1904.00498 [astro-ph.CO]

26. E. Armengaud et al., EDELWEISS Collaboration. Phys. Rev. D 99(8), 082003 (2019). arXiv:1901.03588 [astro-ph.GA]

27. Z.Z. Liu et al., CDEX Collaboration. Phys. Rev. Lett. 123(16), 161301 (2019). arXiv:1905.00354 [hep-ex]

28. J. Aasi et al., LIGO scientific. Class. Quant. Grav. 32, 074001 (2015). arXiv:1411.4547 [gr-qc]

29. LHC Guide, CERN Brochure 2017, http://cds.cern.ch/record/ 2255762. Accessed 28 Apr 2020

30. K. Somiya, KAGRA. Class. Quant. Grav. 29, 124007 (2012). arXiv:1111.7185 [gr-qc]

31. B.P. Abbott et al., LIGO Scientific and Virgo Collaborations. Phys. Rev. Lett. 116(13), 131103 (2016). arXiv:1602.03838 [gr-qc]

32. See, e.g., Secs. 9.5.2 and 9.5.3 of K.S. Thorne, in Three Hundred Years of Gravitation, eds. S.W. Hawking and W. Israel, (Cambridge University Press, Cambridge, 1987), and references therein, ISBN 978-0521379762

33. S. Tsuchida, N. Kanda, Y. Itoh, M. Mori, Phys. Rev. D 101(2), 023005 (2020). arXiv:1909.00654 [astro-ph.HE]

34. C.-H. Lee, C. S. Nugroho, and M. Spinrath, work in progress

35. W.L. Xu, C. Dvorkin, A. Chael, Phys. Rev. D 97(10), 103530 (2018). arXiv:1802.06788 [astro-ph.CO]

36. E.O. Nadler, V. Gluscevic, K.K. Boddy, R.H. Wechsler, Astrophys. J. Lett. 878(2), 32 (2019). arXiv:1904.10000 [astro-ph.CO] 